\title{
Epigenetic inactivation of calcium-sensing receptor in colorectal carcinogenesis
}

\author{
Keiichi Hizaki ${ }^{1,4}$, Hiroyuki Yamamoto ${ }^{1,4}$, Hiroaki Taniguchi ${ }^{1}$, Yasushi Adachi $^{1}$, \\ Mayumi Nakazawa ${ }^{1}$, Tokuma Tanuma ${ }^{1}$, Norihiro Kato ${ }^{1}$, Yasutaka Sukawa ${ }^{1}$, \\ Jose V Sanchez ${ }^{2}$, Hiromu Suzuki ${ }^{1}$, Shigeru Sasaki ${ }^{1}$, Kohzoh Imai ${ }^{3}$ and Yasuhisa Shinomura ${ }^{1}$ \\ ${ }^{1}$ First Department of Internal Medicine, Sapporo Medical University School of Medicine, Sapporo, Japan; \\ ${ }^{2}$ Cancer Epigenetics and Biology Program, Catalan Institute of Oncology, Barcelona, Spain and ${ }^{3}$ The \\ Advanced Clinical Research Center, The Institute of Medical Science, The University of Tokyo, Tokyo, Japan
}

\begin{abstract}
$\mathrm{Ca}^{2+}$ is a chemopreventive agent for colon cancer. Ion transport systems are often altered in human cancer. The aim of this study was to clarify the alterations of calcium-sensing receptor (CASR), a member of the G proteincoupled receptor family, in colorectal carcinogenesis. We analyzed the expression of CASR in colorectal cancer cell lines and in cancer and adenoma tissues by RT-PCR and immunostaining. In addition, we analyzed methylation of the CASR promoter by using bisulfite sequence analysis and methylation-specific PCR. CASR mRNA and protein expression was significantly downregulated in most of the cancer cell lines. CpG islands were densely methylated in cancer cell lines with reduced CASR mRNA expression. Treatment with a demethylating agent, 5-aza-2'-deoxycytidine, and/or a histone deacetylase inhibitor, trichostatin A, restored CASR expression in the cancer cell lines. Disruption of CASR expression in CASR-unmethylated HCT-8 cells blocked the enhancing effect of $\mathrm{Ca}^{2+}$ on the cytotoxic response to 5-fluorouracil. CASR expression was observed in normal colonic epithelial cells and was retained in most adenoma tissues. CASR mRNA and protein expression was significantly downregulated in cancer tissues. There was an inverse relationship between CASR expression and degree of differentiation. Immunohistochemical CASR staining was reduced more predominantly in less-differentiated cancer tissues and/or in cancer cells at the invasive front, where nuclear/ cytoplasmic $\beta$-catenin was often localized. CASR methylation was detected in $69 \%$ of colorectal cancer tissues and $90 \%$ of lymph node metastatic tissues and was significantly correlated with reduced CASR expression. CASR methylation was also detected in $32 \%$ of advanced adenoma tissues but was detected in only $9 \%$ of adenoma tissues and was not detected in hyperplastic polyp tissues. CASR methylation seems to occur at an early stage and progress in colorectal carcinogenesis. The results suggest that epigenetic inactivation of CASR has an important role in colorectal carcinogenesis.
\end{abstract}

Modern Pathology (2011) 24, 876-884; doi:10.1038/modpathol.2011.10; published online 11 February 2011

Keywords: calcium-sensing receptor; chemoprevention; colorectal adenoma; colorectal carcinogenesis; DNA methylation

Colorectal cancer is one of the most common cancers in the world. Various genetic and epigenetic abnormalities have been identified in colorectal cancer, suggesting a multistep nature of colorectal carcinogenesis. ${ }^{1-3} \mathrm{~A}$ genetic predisposition has a role in some individuals with colorectal cancer. Diet is also thought to be a major etiological factor for

Correspondence: Dr H Yamamoto, MD, PhD, First Department of Internal Medicine, Sapporo Medical University School of Medicine, S-1, W-16, Chuo-ku, Sapporo 060-8543, Japan.

E-mail: h-yama@sapmed.ac.jp

${ }^{4}$ These authors contributed equally to this paper.

Received 23 August 2010; revised 1 December 2010; accepted 1

December 2010; published online 11 February 2011 colorectal cancer. Low intake of calcium and/or vitamin $\mathrm{D}$ is suggested to be one of the important risk factors for colorectal cancer.

$\mathrm{Ca}^{2+}$ has chemopreventive activity for colorectal cancer. ${ }^{4,5}$ Calcium-sensing receptor (CASR) is a member of the $\mathrm{G}$ protein-coupled receptor family. Extracellular $\mathrm{Ca}^{2+}$ and CASR serve as a first messenger system in controlling biological function. In addition to the systemic control of $\mathrm{Ca}^{2+}$ homeostasis, CASR has been suggested to have diverse functions, such as regulation of various cellular processes in different cell types.$^{6-9}$ Expression of CASR in colonic 'epithelial cells has been reported. CASR reportedly controls proliferation and differentiation in the human colonic epithelium and colon carcinoma cells. ${ }^{10-18}$ 
Increased thymidylate synthase expression is one the most important mechanisms underlying the resistance to 5-fluorouracil (FU) in colorectal cancer. ${ }^{19,20}$ Interestingly, activation of CASR reportedly downregulates the expression of thymidylate synthase and survivin, and upregulates cellular sensitivity to 5-FU in human colorectal cancer cell lines. ${ }^{15}$ Moreover, it has been reported that vitamin $\mathrm{D}$ mediates its action in human colorectal cancer cells in a CASR-dependent manner and downregulates malignant cell behavior. ${ }^{21}$

Ion transport systems are often altered in human cancer. Downregulation of CASR protein expression has been reported in colorectal cancer tissues. There are only a few reports showing downregulation of immunohistochemical staining of CASR in colorectal cancer tissues. ${ }^{11,12,22}$ However, the numbers of analyzed cases were too small to characterize the alterations of CASR expression in colorectal carcinogenesis. In addition, alterations of CASR expression in colorectal adenoma have not been reported. Moreover, the mechanism by which CASR expression is downregulated in colorectal cancer is not known. It has been reported that $\mathrm{Na}^{+} /$monocarboxylate transport, a product of the $S L C 5 A 8$ gene, is silenced by promoter hypermethylation in colorectal cancer. ${ }^{23}$ If $C A S R$ is inactivated by DNA methylation, reversal of CASR silencing by demethylating agents could be a potential target for effective treatment and/or prevention of colorectal cancer.

These findings made CASR relevant to further investigations in colorectal carcinogenesis. Thus, we systematically analyzed the expression and epigenetic regulation of CASR in colorectal cancer cell lines and tissues and in adenoma tissues.

\section{Materials and methods}

\section{Cell Lines}

Colorectal cancer cell lines, Caco-2, COLO 320DM, DLD-1, LoVo, HT-29, SW480, SW48, SW620, HCT-8, HCT 116, and hepatoma cell line JHH7, were obtained from the Japanese Cancer Research Resources Bank (Tokyo, Japan), Riken Cell Bank (Tokyo), or the American Type Culture Collection (Rockville, MD, USA), and cultured as recommended. Cells were maintained at $37^{\circ} \mathrm{C}$ in an atmosphere of humidified air with $5 \% \mathrm{CO}_{2}$.

\section{Tissue Samples}

Tissue samples were purchased from Genomics Collaborative (Laurel, MD, USA) or obtained from Japanese patients who had undergone surgical treatment or endoscopy. Informed consent was obtained from each patient. Tissue microarray of colorectal adenocarcinoma tissues was purchased from SuperBioChips Laboratories (Seoul, Korea). Advanced adenoma was defined as an adenoma of
$10 \mathrm{~mm}$ or more in diameter and/or an adenoma with high-grade dysplasia. ${ }^{24,25}$ In all, 90 colorectal adenocarcinoma tissues (mean age: 67 years old; 54 male and 36 female; 39 right sided and 51 left sided (distal to the splenic flexure)), 10 lymph node metastatic tissues (mean age: 71 years old; 6 male and 4 female), 28 advanced adenoma tissues (mean age: 56 years old; 15 male and 13 female; 12 right sided and 16 left sided), 22 adenoma tissues (mean age: 54 years old; 12 male and 10 female; 9 right sided and 13 left sided), and 14 hyperplastic polyp tissues (mean age: 53 years old; 8 male and 6 female; 6 right sided and 8 left sided) were analyzed. The sample was processed for pathological examination using hematoxylin and eosin staining for the evaluation of the tumor cell content. Only specimens containing $>80 \%$ tumor cells were used for analysis. The seventh edition of the tumor-nodemetastasis (TNM) system of the American Joint Committee on Cancer and the International Union against Cancer was used for the pathological diagnosis and classification of variables.

\section{Semiquantitative RT-PCR and Real-Time RT-PCR}

Total RNA from cell lines and tissue samples were extracted using an extraction kit (RNeasy Mini kit; Qiagen, Valencia, CA, USA). cDNA was synthesized from $1 \mu \mathrm{g}$ of total RNA using SuperScript III reverse transcriptase (Invitrogen, San Diego, CA, USA) with random hexamers. PCR was carried out using primers specific for the CASR gene and the glyceraldehyde-3-phosphate dehydrogenase (GAPDH) genes. The primer sequences used were $5^{\prime}$-CGGGG TACCTTAAGCACCTACGGCATCTAA- $3^{\prime}$ and $5^{\prime}$-GCT CTAGAGTTAACGCGATCCCAAAGGGCTC- $3^{\prime}$ for $C A$ $S R$, and $5^{\prime}$-TGGACATCAATGAGTGCCTC- $3^{\prime}$ and $5^{\prime}-$ CACATTCTGGTGAGCATTCG- $3^{\prime}$ for GAPDH. GAPDH served as an internal control of the reaction. To perform semiquantitative RT-PCR, the ranges of linear amplification for the CASR gene and for the $G A P D H$ gene were studied by using standard curves. $^{26}$ Thus, the optimal number of PCR cycles was determined. After PCR, the PCR products were electrophoresed in $2 \%$ agarose gels. Real-time RTPCR was carried out by using SYBR green real-time PCR system. The primer sequences used were $5^{\prime}-$ AAGGGGGACATTATCCTTGG-3' ${ }^{\prime}$ and $5^{\prime}$-GCTGGGC TGCTGTTTATCTC-3'. A comparative threshold cycle $\left(C_{\mathrm{T}}\right)$ was used to determine gene expression relative to the control (calibrator). All reactions were carried out at least in duplicate and controlled without reverse transcriptase.

\section{Bisulfite Sequencing and MSP}

Bisulfite modification of genomic DNA was performed using a Methylamp DNA modification kit (Epigentek, Brooklyn, NY, USA). In the chemical modification of cytosine to uracil by bisulfite 
treatment, all cytosines are converted to uracil, but those that are methylated are resistant to this modification and remain as cytosine. Bisulfite sequencing was carried out ${ }^{27}$ using primers, $5^{\prime}$-TG TTAGGGGTTAGGGATAAGG- $3^{\prime}$ and $5^{\prime}$-ACTCATTC TACAAAACTCAAATCAAA- $3^{\prime}$, designed to amplify the CASR promoter region. MSP analysis was carried out using primers corresponding to the CASR promoter region sequences. One can design PCR primers to distinguish methylated from unmethylated DNA in bisulfite modified DNA, taking advantage of the sequence differences resulting from bisulfite modification. Sequences of methylation-specific primers were 5'-GGTTCGGGTTTT TAAGTAGC- $3^{\prime}$ and $5^{\prime}$-TCAAACGTTACCTATACC GC-3'. Sequences of unmethylation-specific primers were $5^{\prime}$-TTAGGTTTGGGTTTTTAAGTAGT- ${ }^{\prime}$ and $5^{\prime}$-TC AAACATTACCTATACCACAAA-3' . After PCR, the PCR products were electrophoresed in $2 \%$ agarose gels.

\section{5-Aza-2'-Deoxycytidine (5-aza-dC) and/or Trichostatin A Treatment}

To examine the role of $\mathrm{CpG}$ methylation and histone deacetylation in silencing of CASR, cancer cells were treated with $1 \mu \mathrm{M}$ of 5-aza-dC (Sigma, St Louis, MO, USA) for $72 \mathrm{~h}$ or with $100 \mathrm{nM}$ of trichostatin A for $24 \mathrm{~h}$. Cells were also treated with $1 \mu \mathrm{M}$ of 5-aza$\mathrm{dC}$ for $72 \mathrm{~h}$ followed by $100 \mathrm{nM}$ of trichostatin A for an additional $24 \mathrm{~h}$. The timing and sequencing of 5-aza-dC and/or trichostatin A was based on similar preliminary studies as well as published studies. ${ }^{28}$ After the treatment, expression of CASR was analyzed by RT-PCR and immunoblotting.

\section{Immunoblotting}

Immunoblotting for CASR was carried out as described previously. ${ }^{29,30}$ Cell lines were homogenized in lysis buffer,(50mm Tris-HCl (pH 7.4) 1\% Triton X-100, $1 \mathrm{~mm}$ diethyldithiocarbamic acid, $1 \mathrm{mM}$ EDTA, 1\% Tween 20, $10 \mu \mathrm{M}$ leupeptin, and $1 \mathrm{mM}$ phenylmethylsulfonyl fluoride) on ice. After sonication and centrifugation $\left(10000 \mathrm{~g}\right.$ at $\left.4{ }^{\circ} \mathrm{C}\right)$, protein content of the supernatant was measured by the method of Bradford. Solubilized proteins $(100 \mu \mathrm{g})$ were separated by SDS-PAGE and transferred to a polyvinylidene difluoride transfer membrane. After being blocked with $5 \%$ blocking agent, the membrane was incubated with an anti-human CASR rabbit polyclonal antibody (Millipore, Billerica, MA, USA). The protein was visualized using the enhanced chemiluminescence detection system (Amersham Biosciences, UK), and the membranes were stripped and reprobed with mouse anti-actin monoclonal antibody (Thermo Fisher Scientific, Waltham, MA, USA). Results were analyzed using a multiimage analyzer (Bio-Rad, Richmond, CA, USA).

\section{Development of CASR Short Hairpin RNA (shRNA) Stable Transfectants}

CASR shRNA (CASR-shRNA) and control shRNA (Ctrl-shRNA) plasmids were purchased from Santa Cruz Biotechnology (Santa Cruz Biotechnology, CA, USA). Plasmids were transfected into CASR-unmethylated HCT-8 cells using shRNA Plasmid Transfection Reagent (Santa Cruz Biotechnology) according to the manufacturer's protocol. Stable CASR-shRNA or Ctrl-shRNA transfectants were established by selection of cells with puromycin. Clones of stable transfectants were obtained by the use of cloning cylinders.

\section{Cytotoxicity Assays}

HCT8 cells and transfectants were maintained in $\mathrm{Ca}^{2+}$-free Spinner-modified minimal essential medium (Sigma) supplemented with sodium bicarbonate and $5 \%$ fetal bovine serum as described previously. ${ }^{11,12,15}$ To assess the effect of $\mathrm{Ca}^{2+}$ on the cytotoxic response to drugs, the medium of cells was replenished with a medium containing physiological concentrations of $\mathrm{Ca}^{2+}(1.4 \mathrm{mM})$ and 5-FU $(15 \mu \mathrm{M})$. The medium of a parallel set of cultures was replenished with a medium only containing 5 -FU without the addition of exogenous $\mathrm{Ca}^{2+}$. After the treatment for 24 or $48 \mathrm{~h}$, WST- 8 assay was used to determine the cytotoxic response to 5-FU. WST-8 assay was carried out using a Cell Counting Kit-8 (Dojindo, Kumamoto, Japan) according to the manufacturer's instructions.

\section{Immunohistochemistry}

Sections ( $5 \mu \mathrm{m}$ thick) of formalin-fixed and paraffinembedded tissue and tissue microarray were dewaxed in xylene and rehydrated in alcohol. The sections were then heated to $105{ }^{\circ} \mathrm{C}$ in target retrieval solution (DakoCytomation, Carpinteria, CA, USA) in an autoclave for $10 \mathrm{~min}$ for antigen retrieval. Endogenous peroxidase activity was suppressed using a solution of $3 \%$ hydrogen peroxide in methanol for $5 \mathrm{~min}$. After being rinsed twice in phosphate-buffered saline (PBS), the sections were incubated for $18 \mathrm{~h}$ at $4{ }^{\circ} \mathrm{C}$ with an anti-human CASR rabbit polyclonal antibody $(1 / 100$, abcam, Cambridge, MA, USA) or an anti-human $\beta$-catenin monoclonal antibody (Santa Cruz Biotechnology). ${ }^{31}$ The antibodies were diluted in antibody diluent with background reducing components $(0.05 \mathrm{~mol} / \mathrm{l}$ Tris-HCl buffer containing $0.1 \%$ Tween and $0.015 \mathrm{~mol} / \mathrm{l}$ sodium azide, DakoCytomation). Normal rabbit or mouse immunoglobulins were substituted for each primary antibody as negative controls. After washing three times in PBS, the sections were treated with biotinylated goat anti-rabbit or antimouse immunoglobulin (DakoCytomation) for $10 \mathrm{~min}$ and then with horseradish peroxidaseavidin complex, diluted as recommended by the 
manufacturer, for $10 \mathrm{~min}$. The slides were then washed in PBS and developed in $0.05 \mathrm{~mol} / \mathrm{l}$ Tris-

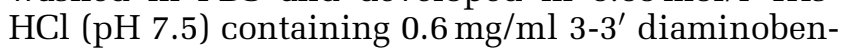
zidine at room temperature. The sections were counterstained in Mayer's hematoxylin and mounted. Cancer cases were categorized into the following three groups corresponding to immunostaining patterns of $\beta$-catenin: membranous, membranous staining pattern similar to that in normal colon mucosa; weak, no staining or weaker staining than normal colon mucosa; and nuclear/cytoplasmic, diffuse staining in the nucleus and/or cytoplasm. When a cancer contained multiple staining patterns, the case was categorized into the dominant staining pattern.

\section{Statistical Analysis}

The results were assessed for associations with clinicopathological parameters using the following statistical tests: Student's $t$-test for age, the MannWhitney test for lymph node metastasis and pTNM stage, and the $\chi^{2}$-test or Fisher's exact test for the remaining parameter. A $P$-value of $<0.05$ was considered significant. A $P$-value between 0.05 and 0.10 was considered as trend toward an association.

\section{Results}

\section{Reduced Expression of CASR mRNA in Colorectal Cancer Cell Lines}

We examined CASR mRNA expression in cancer cell lines by using semiquantitative RT-PCR (Figure 1). The CASR mRNA expression was frequently lost or considerably reduced in cancer cell lines (Figure 1). There was no correlation between CASR expression and histopathology. Concordant results were obtained by real-time RT-PCR (data not shown).

\section{Bisulfite Sequencing and MSP of the CASR Promoter in Colorectal Cancer Cell Lines}

Methylation status of the CpG islands of CASR was analyzed in colorectal cancer cell lines. The bisulfite

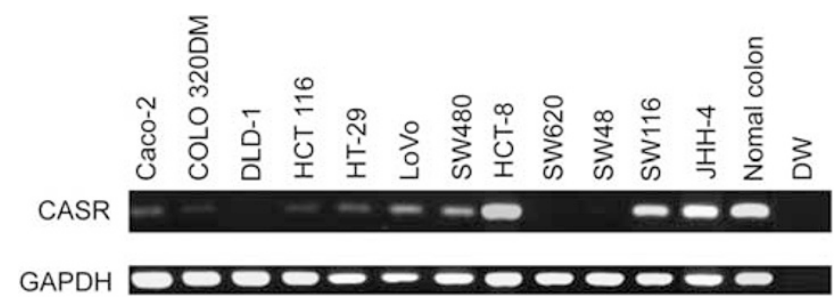

Figure 1 Expression of CASR mRNA in colorectal cancer cell lines analyzed by semiquantitative RT-PCR analysis. RT-PCR was carried out using cDNA from 11 colorectal cancer cell lines. $G A P D H$ served as a control. The CASR mRNA expression was frequently lost or considerably reduced in cancer cell lines. sequencing method was used to analyze the methylation status of $11 \mathrm{CpG}$ sites in the 218-bp fragment of the CASR promoter including the transcriptional start site (Figure 2a). These CpG islands were densely methylated in cancer cell lines with no or reduced CASR mRNA expression but not in the HCT-8 cell line expressing CASR. Methylation status of $C A S R$ was further analyzed in cancer cell lines by using MSP (Figure 2b). A methylated PCR product was detected in cell lines with no or reduced $C A S R$ mRNA expression. Only an unmethylated PCR product was detected in the HCT-8 cell line expressing CASR. Thus, downregulation of CASR mRNA expression was correlated with hypermethylation of the promoter.

\section{Reactivation of CASR Expression by 5-aza-dC/ trichostatin A Treatment in Colorectal Cancer Cell Lines}

To further examine the role of $\mathrm{CpG}$ methylation and histone deacetylation in silencing of CASR, cancer cells were treated with 5-aza-dC and/or trichostatin A. Treatment with 5-aza-dC restored CASR expression, and combined treatment with 5-aza-dC and trichostatin A restored CASR expression synergistically at mRNA and protein levels in colorectal cancer cell lines (Figure 3).

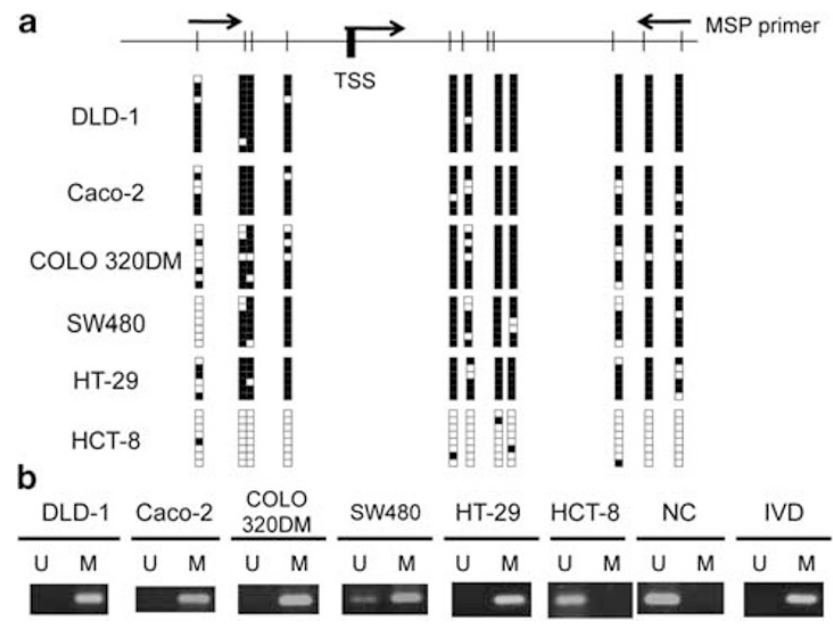

Figure 2 Bisulfite sequencing (a) and MSP (b) analyses of CASR promoter in colorectal cancer cell lines. Methylation status of the CpG islands of CASR was analyzed in colorectal cancer cell lines. (a) The bisulfite sequencing method was used to analyze methylation status of $11 \mathrm{CpG}$ sites in the 218-bp fragment of the $C A S R$ promoter including the transcriptional start site. Unmethylated CpG sites are indicated by open boxes and methylated CpG sites are indicated by filled boxes. These CpG islands were densely methylated in cancer cell lines with no or reduced CASR mRNA expression but not in the HCT-8 cell line expressing CASR. The arrows represent primers for MSP. (b) Methylation status of CASR was further analyzed in cancer cell lines by using MSP. NC, normal colorectal mucosa; IVD, in vitro methylated DNA. A methylated PCR product was detected in cell lines with no or reduced CASR mRNA expression. Only an unmethylated PCR product was detected in the HCT-8 cell line expressing CASR. 


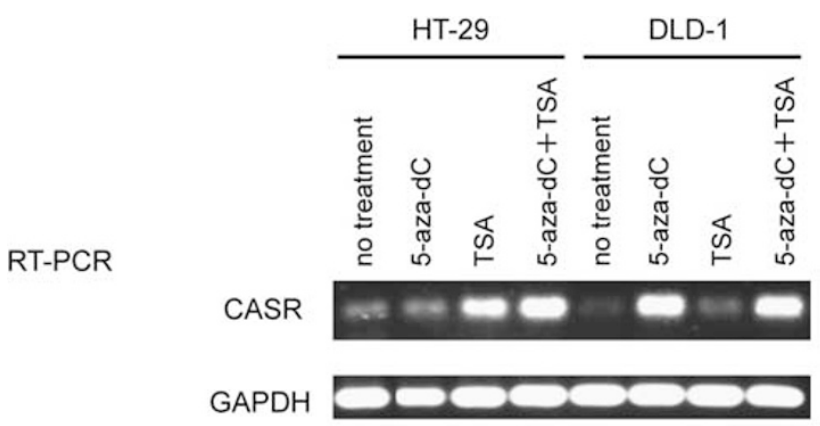

Immunoblotting

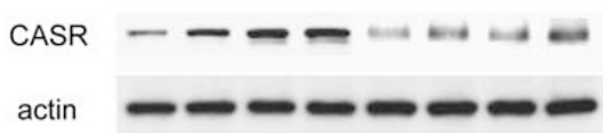

Figure 3 Reactivation of CASR by 5-aza-dC and/or trichostatin A treatment in colorectal cancer cell lines. To examine the role of CpG methylation and histone deacetylation in silencing of CASR, cancer cells were treated with 5 -aza-dC and/or trichostatin A. As indicated, cells were incubated for $72 \mathrm{~h}$ with $1 \mu \mathrm{M} 5$-aza-dC and/or for $24 \mathrm{~h}$ with $100 \mathrm{nM}$ trichostatin A. After the treatment, expression of CASR was analyzed by RT-PCR and immunoblotting. Treatment with 5-aza-dC restored CASR expression, and combined treatment with 5 -aza-dC and trichostatin A restored CASR expression synergistically at mRNA and protein levels in colorectal cancer cell lines.

Block of the Enhancing Effect of $\mathrm{Ca}^{2+}$ on the Cytotoxic Response to 5-FU by Disruption of CASR Expression

shRNA approach was used to target the CASR to determine the role of CASR in mediating the action of extracellular $\mathrm{Ca}^{2+}$. Expression of CASR was significantly downregulated in CASR shRNA-transfected HCT-8 cells, but not in control shRNAtransfected cells (data not shown). After treatment with 5-FU for $24 \mathrm{~h}$, extracellular $\mathrm{Ca}^{2+}$ enhanced the sensitivity to 5-FU in control shRNA-transfected HCT-8 cells, but not in CASR shRNA-transfected HCT-8 cells (Figure 4). After treatment with 5-FU for $48 \mathrm{~h}$, similar results were obtained (data not shown).

\section{Reduced Expression of CASR mRNA in Colorectal Tumor Tissues}

Downregulation of CASR mRNA expression was observed in $24(60 \%)$ of 40 cancer tissues compared with expression in their matched normal tissues (Figure 5 and data not shown). Reduced mRNA expression of $C A S R$ was observed in $21(70 \%)$ of 30 pT2-pT4 cancer tissues and in $3(30 \%)$ of 10 pT1 cancer tissues. Reduced mRNA expression of CASR was also observed in $4(21 \%)$ of 14 advanced adenoma tissues but in only one (8\%) of 12 adenoma tissues and in none of 10 hyperplastic polyp tissues (data not shown).

\section{Expression of CASR and $\beta$-Catenin Protein in Colorectal Tumor Tissues}

Figure 6 shows representative results of immunohistochemistry for CASR and $\beta$-catenin in colorectal

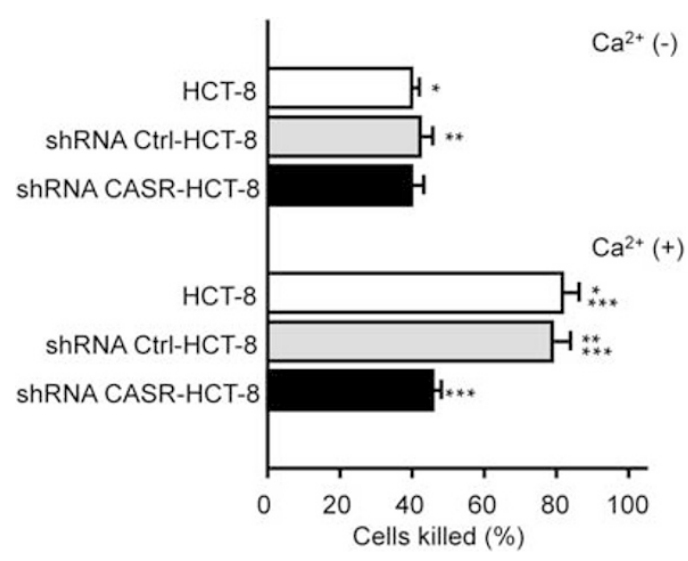

Figure 4 Block of the enhancing effect of $\mathrm{Ca}^{2+}$ on the cytotoxic response to 5-FU by disruption of CASR expression. shRNA approach was used to target the CASR to determine the role of CASR in mediating the action of extracellular $\mathrm{Ca}^{2+}$. Parental cells and transfectants were exposed to $5-\mathrm{FU}$ for $24 \mathrm{~h}$ and their cytotoxic response to 5-FU was evaluated by using WST-8 assay. $*, * *$, and $* * *$ indicate $P<0.01$. After treatment with 5 -FU for $24 \mathrm{~h}$, extracellular $\mathrm{Ca}^{2+}$ enhanced the sensitivity to 5 -FU in control shRNA-transfected HCT-8 cells, but not in CASR shRNAtransfected HCT-8 cells.

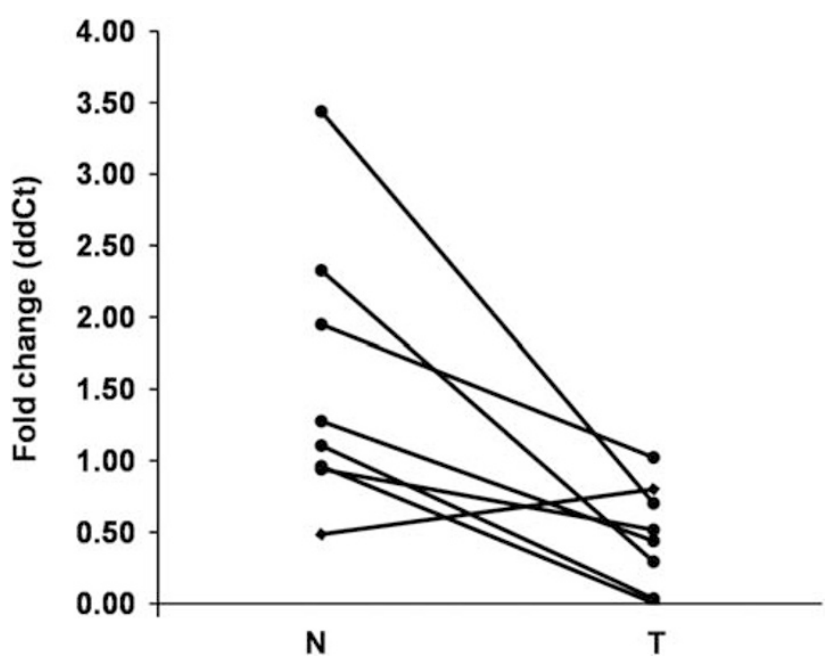

Figure 5 Expression of CASR mRNA in colorectal cancer tissues analyzed by real-time RT-PCR analysis. Real-time RT-PCR was carried out by using SYBR Green real-time PCR system. N and T, matched samples from non-tumor and tumor tissue, respectively. CASR mRNA expression was reduced in seven of eight cancer tissues compared with expression in their matched normal tissues.

cancer tissues. There was no detectable immunoreactivity with the control normal rabbit or mouse immunoglobulins (data not shown). CASR was stained in columnar epithelial cells of normal colonic crypts and used as positive controls. In 51 $(57 \%)$ of 90 cancer tissues, CASR expression in cancer cells was weak or lost when compared with that in adjacent normal colonic epithelial cells in the same section. Reduced CASR expression was observed in $45(64 \%)$ of 70 pT2-pT4 cancer tissues and in $6(30 \%)$ of 20 pT1 cancer tissues. 

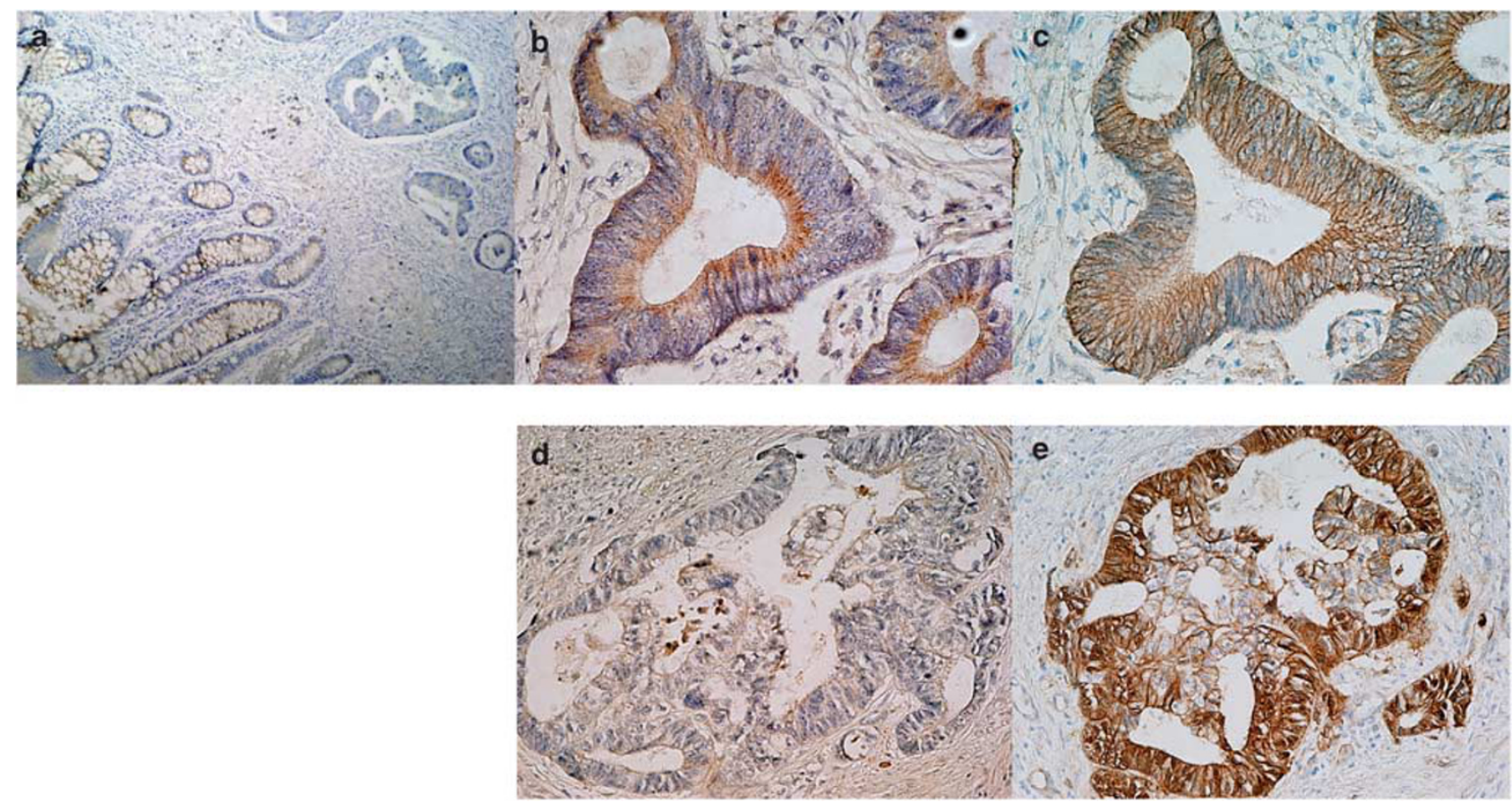

Figure 6 Immunohistochemistry for CASR and $\beta$-catenin in colorectal cancer tissues. Immunohistochemistry with an anti-human CASR rabbit polyclonal antibody and an anti-human $\beta$-catenin monoclonal antibody was carried out in 90 colorectal cancer tissues. (a) Normal epithelial tissues positive for CASR (left) and cancer cells negative for CASR (right), (b) cancer tissue positive for CASR, (c) cancer tissue with membranous $\beta$-catenin staining. (d) Cancer tissue negative for CASR. In 51 (57\%) of 90 cancer tissues, CASR expression in cancer cells was weak or lost when compared with that in adjacent normal colonic epithelial cells. (e) Cancer tissue with nuclear/cytoplasmic $\beta$-catenin staining. Original magnification: $\mathbf{a}, \times 100$; b-e, $\times 400$. CASR expression was reduced more predominantly in lessdifferentiated cancer tissues or in cancer cells at the invasive front, where nuclear/ cytoplasmic $\beta$-catenin was often localized. Nuclear/ cytoplasmic $\beta$-catenin expression was more frequently observed in cases with reduced CASR expression than in cases with normal CASR expression $(P<0.01)$.

Downregulation of CASR expression was inversely correlated with degree of differentiation $(P<0.01)$. Membranous staining of $\beta$-catenin was observed in normal epithelium and used as positive controls. CASR expression was reduced more predominantly in less-differentiated cancer tissues or in cancer cells at the invasive front, where nuclear/cytoplasmic $\beta$-catenin was often localized. Nuclear/cytoplasmic $\beta$-catenin expression was more frequently observed in cases with reduced CASR expression than in cases with normal CASR expression $(P<0.01)$. Reduced expression of CASR was observed in $6(21 \%)$ of 28 advanced adenoma tissues but only in $2(9 \%)$ of 22 adenoma tissues and in none of 14 hyperplastic polyp tissues (data not shown).

\section{CASR Methylation in Colorectal Tumor Tissues}

CASR methylation status was analyzed in colorectal tumor tissues (Figure 7). CASR methylation was detected in $62(69 \%)$ of 90 cancer tissues (Figure 7a) and it was inversely correlated with degree of differentiation $(P<0.01)$. CASR methylation was significantly correlated with reduced mRNA and protein expression of CASR $(P<0.01)$. CASR methylation was detected in $53(76 \%)$ of 70 pT2-pT4 cancer tissues, 9 (45\%) of 20 pT1 cancer tissues, and 9 (90\%) of 10 lymph node metastatic tissues. CASR methylation was detected in $9(32 \%)$ of 28 advanced adenoma tissues but only in $2(9 \%)$ of 22 adenoma tissues and in none of 14 hyperplastic polyp tissues (Figure $7 \mathrm{~b}$ and $\mathrm{c}$ ).

\section{Discussion}

In this study, we demonstrated frequent downregulation of CASR mRNA expression in colorectal cancer cell lines including moderately differentiated ones. As a mechanism of CASR downregulation in cancer, we speculated that CASR may be silenced by promoter hypermethylation. By using bisulfite sequence analysis and MSP, we demonstrated that silencing of $C A S R$ was correlated with hypermethylation of the promoter in colorectal cancer cell lines. To confirm the role of epigenetic alterations in transcriptional repression of the CASR gene, we treated colorectal cancer cell lines, in which CASR was methylated, with 5-aza-dC alone or in combination with trichostatin A. Treatment with 5-aza-dC restored the CASR expression in cancer cell lines. Moreover, combined treatment with 5-aza-dC and trichostatin A restored CASR expression synergistically, indicating that $\mathrm{CpG}$ methylation and histone 


$\frac{1 N}{U M} \frac{1 T}{U M} \frac{2 N}{U M} \frac{2 T}{U M} \frac{3 T}{U M} \frac{4 T}{U M} \frac{5 T}{U M} \frac{6 T}{U M} \frac{7 T}{U M}$
$\frac{8 T}{U M} \frac{9 T}{U M} \frac{10 T}{U M} \frac{L M 1}{U M} \frac{L M 2}{U M} \frac{L M 3}{U M} \frac{L M 4}{U M} \frac{N L}{U M} \frac{I V D}{U M}$

b

$\frac{A A 1}{U M} \frac{A A 2}{U M} \frac{A A 3}{U M} \frac{A A 4}{U M} \frac{A D}{U M} \frac{A D 2}{U M} \frac{A D}{U M}$
$\frac{A D 4}{U M} \frac{H P 1}{U M} \frac{H P 2}{U M} \frac{H P 3}{U M} \frac{H P 4}{U M} \frac{N C}{U M} \frac{I V D}{U M}$
$-M$

C

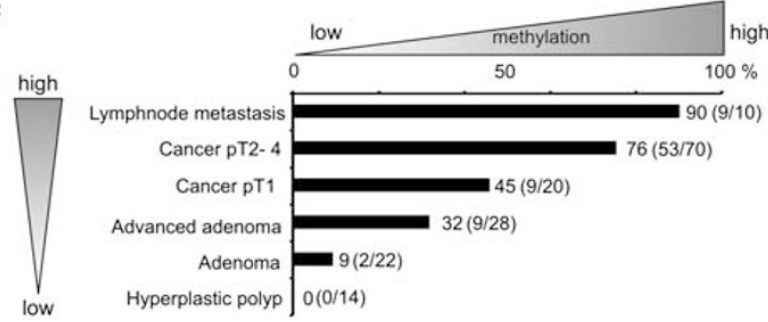

Figure 7 MSP analysis of CASR in colorectal tumor tissues. CASR methylation status was analyzed in colorectal tumor tissues by MSP. (a) CASR methylation status in normal (N), cancer (T), and lymph node metastatic (LM) tissues. $\mathrm{U}$ and $\mathrm{M}$, unmethylated and methylated PCR products, respectively. NL, normal lymphocytes; IVD, in vitro methylated DNA. In total, CASR methylation was detected in $62(69 \%)$ of 90 cancer tissues and it was inversely correlated with degree of differentiation $(P<0.01)$. (b) $C A S R$ methylation status in advanced adenoma (AA), adenoma (AD), and hyperplastic polyp (HP) tissues. U and M, unmethylated and methylated PCR products, respectively. NC, normal colorectal mucosa. In total, CASR methylation was detected in $9(32 \%)$ of 28 advanced adenoma tissues but only in $2(9 \%)$ of 22 adenoma tissues and in none of 14 hyperplastic polyp tissues. (c) Frequency of $C A S R$ methylation in colorectal tumor tissues. Progressive epigenetic silencing of CASR was observed in colorectal carcinogenesis.

deacetylation has an important role in silencing the CASR gene.

We showed that extracellular $\mathrm{Ca}^{2+}$ enhanced the cytotoxic response of CASR-unmethylated HCT-8 cells to 5-FU. This enhancing effect of extracellular $\mathrm{Ca}^{2+}$ required a functional CASR because knocking down CASR expression abrogated the effect of extracellular $\mathrm{Ca}^{2+}$.

The results of in vitro studies were further supported by results for primary tumor tissues. CASR mRNA expression in cancer cells was frequently downregulated in colorectal cancer tissues including pT1 cases. Downregulation of CASR expression was also observed at the protein level analyzed by immunohistochemistry. A significant inverse association was found between CASR downregulation and degree of differentiation. Moreover, CASR mRNA and protein expression was downregulated in advanced adenoma tissues at a lower frequency than that in cancer tissues. In contrast,
CASR expression was retained in adenoma tissues and hyperplastic polyp tissues. These results suggest that downregulation of CASR expression occurs in the early stage and progresses in colorectal carcinogenesis.

One of the supposed functions of extracellular $\mathrm{Ca}^{2+}$ and the CASR in colorectal cancer cells is suppression of $\beta$-catenin/T-cell factor activation. ${ }^{11,14}$ In a small series of colorectal cancer tissues $(n=6)$, a relationship between CASR expression and $\beta$-catenin staining pattern was reported. ${ }^{14}$ In this study, nuclear/cytoplasmic $\beta$-catenin expression was more frequently observed in cases with reduced CASR expression than in cases with normal CASR expression. As various genetic and epigenetic alterations are involved in $\beta$-catenin/T-cell factor activation, the relationship between $\beta$-catenin expression and CASR expression seems not to be simple. Further analyzes are needed to address these issues.

By using MSP, we detected CASR methylation in $69 \%$ of cancer tissues and it was inversely correlated with degree of differentiation. CASR methylation was significantly correlated with reduced mRNA and protein expression of CASR. Interestingly, CASR methylation was detected in $90 \%$ of lymph node metastatic tissues. These results suggest that CASR downregulation by DNA methylation contributes to abnormal differentiation and malignant progression. Moreover, CASR methylation was detected in $32 \%$ of advanced adenoma tissues but was detected in only in $9 \%$ of adenoma tissues and was not detected in hyperplastic polyp tissues. CASR methylation in advanced adenoma tissues was less significantly correlated with reduced protein expression, suggesting monoallelic and/or heterogeneous methylation. These results further support the notion that progressive epigenetic silencing of CASR has an important role in colorectal carcinogenesis.

Activation of CASR reportedly downregulates the expression of thymidylate synthase and survivin, and upregulates cellular sensitivity to 5-FU in human colon carcinoma cells. ${ }^{15}$ Liu et $a l^{15}$ reported that functional CASR is necessary for tumor suppressive function of vitamin $\mathrm{D}$ and that this function is abrogated by knocking down CASR expression. Therefore, from the viewpoint of chemotherapy and/ or chemoprevention using $\mathrm{Ca}^{2+}$ and/or vitamin $\mathrm{D}$, the results obtained in this study are important. If the CASR gene is methylated, reversal of CASR silencing by demethylating agents might be a potential target for effective treatment and/or prevention of colorectal cancers. Calcimimetics enhance CASR agonist activity. ${ }^{16}$ The use of calcimimetics might be beneficial in cases characterized by reduced CASR levels. However, systemic use of allosteric agonists of the CASR might be associated with potentially major adverse effects. ${ }^{16}$ Development of organ- or tissue-specific targeting methods will be necessary to avoid these adverse effects. 
Hypermethylation of the CASR gene may provide a useful marker for detecting preinvasive and early stage cancer amenable to cure and/or for predicting the risk of cancer. Analysis of DNA methylation and/or expression of CASR in precancerous or early carcinoma lesions may enable prediction of which lesions will respond to the differentiation-inducing or chemopreventive activity of extracellular $\mathrm{Ca}^{2+}$ and/or vitamin $\mathrm{D}$.

\section{Acknowledgements}

This work was supported by Grants-in-Aid for Scientific Research from the Ministry of Education, Culture, Sports, Science, and Technology of Japan (HY, KI, and YS) and supported in part by program for developing the supporting system for upgrading the education and research from the Ministry of Education, Culture, Sports, Science, and Technology (YS).

\section{Disclosure/conflict of interest}

The authors declare no conflict of interest.

\section{References}

1 Markowitz SD, Bertagnolli MM. Molecular origins of cancer: molecular basis of colorectal cancer. N Engl J Med 2009;361:2449-2460.

2 Imai K, Yamamoto H. Carcinogenesis and microsatellite instability: the interrelationship between genetics and epigenetics. Carcinogenesis 2008;29:673-680.

3 Slaby O, Svoboda M, Michalek J, et al. MicroRNAs in colorectal cancer: translation of molecular biology into clinical application. Mol Cancer 2009;8:102-114.

4 Lipkin M. Preclinical and early human studies of calcium and colon cancer prevention. Ann N Y Acad Sci 1999;889:120-127.

5 Wargovich MJ, Jimenez A, McKee K, et al. Efficacy of potential chemopreventive agents on rat colon aberrant crypt formation and progression. Carcinogenesis 2000;21:1149-1155.

6 Hhebert SC, Brown EM. The extracellular calcium receptor. Curr Opin Cell Biol 1995;7:484-492.

7 McNeil SE, Hobson SA, Nipper V, et al. Functional calcium-sensing receptors in rat fibroblasts are required for activation of src kinase and mitogenactivated protein kinase in response to extracellular calcium. J Biol Chem 1998;273:1114-1120.

8 Rutten MJ, Bacon KD, Marlink KL, et al. Identification of a functional Ca2+-sensing receptor in normal human gastric mucous epithelial cells. Am J Physiol 1999;277:G662-G670.

9 Geibel JP, Hebert SC. The functions and roles of the extracellular $\mathrm{Ca} 2+$-sensing receptor along the gastrointestinal tract. Annu Rev Physiol 2009;71:205-217.

10 Brown EM, Pollak M, Hebert SC. The extracellular calcium-sensing receptor: its role in health and disease. Annu Rev Med 1998;49:15-29.

11 Chakrabarty S, Radjendirane V, Appelman $\mathrm{H}$, et al. Extracellular calcium and calcium sensing receptor function in human colon carcinomas: promotion of Ecadherin expression and suppression of beta-catenin/ TCF activation. Cancer Res 2003;63:67-71.

12 Chakrabarty $\mathrm{S}$, Wang $\mathrm{H}$, Canaff $\mathrm{L}$, et al. Calcium sensing receptor in human colon carcinoma: interaction with $\mathrm{Ca}^{2+}$ and 1,25-dihydroxyvitamin D3. Cancer Res 2005;65:493-498.

13 Bhagavathula N, Kelley EA, Reddy M, et al. Upregulation of calcium-sensing receptor and mitogen-activated protein kinase signalling in the regulation of growth and differentiation in colon carcinoma. $\mathrm{Br} \mathrm{J}$ Cancer 2005;93:1364-1371.

14 Bhagavathula N, Hanosh AW, Nerusu KC, et al. Regulation of E-cadherin and beta-catenin by $\mathrm{Ca}^{2+}$ in colon carcinoma is dependent on calcium-sensing receptor expression and function. Int $\mathrm{J}$ Cancer 2007;121:1455-1462.

15 Liu G, Hu X, Varani J, et al. Calcium and calcium sensing receptor modulates the expression of thymidylate synthase, NAD(P)H:quinone oxidoreductase 1 and survivin in human colon carcinoma cells: promotion of cytotoxic response to mitomycin $\mathrm{C}$ and fluorouracil. Mol Carcinog 2009;48:202-211.

16 Saidak Z, Mentaverri R, Brown EM. The role of the calcium-sensing receptor in the development and progression of cancer. Endocr Rev 2009;30:178-195.

17 Whitfield JF. Calcium, calcium-sensing receptor and colon cancer. Cancer Lett 2009;275:9-16.

18 Ghevariya V, Anand S. A short primer on the calcium sensing receptor: an important cog in the colon cancer wheel? Dig Dis Sci 2011;56:279-284.

19 Rose MG, Farrell MP, Schmitz JC. Thymidylate synthase: a critical target for cancer chemotherapy. Clin Colorectal Cancer 2002;1:220-229.

20 Chu E, Callender MA, Farrell MP, et al. Thymidylate synthase inhibitors as anticancer agents: from bench to bedside. Cancer Chemother Pharmacol 2003;52: S80-S89.

21 Liu G, Hu X, Chakrabarty S. Vitamin D mediates its action in human colon carcinoma cells in a calciumsensing receptor-dependent manner: downregulates malignant cell behavior and the expression of thymidylate synthase and survivin and promotes cellular sensitivity to 5-FU. Int J Cancer 2010;126:631-639.

22 Sheinin Y, Kállay E, Wrba F, et al. Immunocytochemical localization of the extracellular calcium-sensing receptor in normal and malignant human large intestinal mucosa. J Histochem Cytochem 2000;48: 595-602.

23 Li H, Myeroff L, Smiraglia D, et al. SLC5A8, a sodium transporter, is a tumor suppressor gene silenced by methylation in human colon aberrant crypt foci and cancers. Proc Natl Acad Sci USA 2003;100:8412-8417.

24 Imperiale TF, Ransohoff DF, Itzkowitz SH, et al. Fecal DNA vs fecal occult blood for colorectal-cancer screening in an average-risk population. $\mathrm{N}$ Engl J Med 2004;351:2704-2714.

25 Morikawa T, Kato J, Yamaji Y, et al. A comparison of the immunochemical fecal occult blood test and total colonoscopy in the asymptomatic population. Gastroenterology 2005;129:422-428.

26 Taniguchi H, Yamamoto H, Hirata T, et al. Frequent epigenetic inactivation of Wnt inhibitory factor-1 in human gastrointestinal cancers. Oncogene 2005;24: 7946-7952.

27 Taniguchi H, Yamamoto H, Akutsu N, et al. Transcriptional silencing of hedgehog interacting protein by 
CpG hypermethylation and chromatic structure in human gastrointestinal cancer. J Pathol 2007;213: 131-139.

28 Cameron EE, Bachman KE, Myohanen S, et al. Synergy of demethylation and histone deacetylase inhibition in the re-expression of genes silenced in cancer. Nat Genet 1999;21:103-107.

29 Maehata T, Taniguchi H, Yamamoto H, et al. Transcriptional silencing of Dickkopf gene family by $\mathrm{CpG}$ island hypermethylation in human gastrointestinal cancer. World J Gastroenterol 2008;14:2710-2722.

30 Takagi H, Sasaki S, Suzuki H, et al. Frequent epigenetic inactivation of SFRP genes in hepatocellular carcinoma. J Gastroenterol 2008;43:378-389.

31 Hirata T, Yamamoto H, Taniguchi H, et al. Characterization of immune escape phenotype of human gastric cancers with and those without high-frequency microsatellite instability. J Pathol 2007;211:516-523. 